\title{
Stimuli-Responsive Hydrogels Based on Polyglycerol Crosslinked with Citric and Fatty Acids
}

\author{
Leidy C. Solano-Delgado ${ }^{(D},{ }^{1}$ César A. Bravo-Sanabria $\mathbb{D}^{1,2}$ \\ Carolina Ardila-Suárez, ${ }^{1,2}$ and Gustavo E. Ramírez-Caballero (iD) \\ ${ }^{1}$ Grupo de Investigación en Polímeros, Escuela de Ingeniería Química, Universidad Industrial de Santander (UIS), \\ Bucaramanga, Colombia \\ ${ }^{2}$ Centro de Investigaciones en Catálisis, Escuela de Ingeniería Química, Universidad Industrial de Santander (UIS), \\ Bucaramanga, Colombia
}

Correspondence should be addressed to Gustavo E. Ramírez-Caballero; gusramca@uis.edu.co

Received 26 October 2017; Accepted 24 December 2017; Published 1 February 2018

Academic Editor: Junshi Zhang

Copyright (C) 2018 Leidy C. Solano-Delgado et al. This is an open access article distributed under the Creative Commons Attribution License, which permits unrestricted use, distribution, and reproduction in any medium, provided the original work is properly cited.

Polyglycerol-based hydrogels from biodegradable raw materials were synthesized by crosslinking reactions of polyglycerol with citric and fatty acids. Three hydrogels were studied varying molar ratios of crosslinking agent. It was found that crosslink amount, type, and size play a crucial role in swelling, thermal, mechanical, and stimuli-responsive properties. The hydrogels absorption capacity changed in response to temperature and $\mathrm{pH}$ external stimuli. The hydrogel with the highest swelling capacity absorbed more than 7 times its own weight at room temperature and $\mathrm{pH}$. This material increased 14 times its own weight at $\mathrm{pH}$ 10. Creep-recovery tests were performed to study the effect of crosslinking agent on mechanical properties. Deformation and percentage of recovery of synthesized hydrogels were obtained. Formation of hydrogels was confirmed using FTIR, and physicochemical properties were analyzed by Scanning Electron Microscopy (SEM), Differential Scanning Calorimetric (DSC), and Dynamic Mechanical Analysis (DMA). This paper aims to give a contribution to biobased hydrogel knowledge from chemical, physicochemical, and mechanical point of view.

\section{Introduction}

Hydrogels are polymeric crosslinked, hydrophilic, threedimensional networks that are not soluble in water but can absorb large quantities of this molecule $[1,2]$. Due to their swelling ability, hydrogels have been studied extensively for a variety of applications such as drug delivery [3-6], agricultural applications $[7,8]$, removal of impurities in aqueous solutions $[9,10]$, biosensors [11], and spectrophotometric determination of drugs [12]. In these applications, swelling capacity and thermal, mechanical, and stimuli-responsive properties are of most interest. These properties are prescribed by the intrinsic properties of the main chain polymer and the crosslinking characteristics, such as type, amount, and size of crosslinking molecules, as well as environmental conditions. In this work, synthesized hydrogels are based on renewable materials; the main chain polymer is polyglycerol, obtained by glycerol polymerization [13], and citric and fatty acids are the crosslinking molecules. Polyglycerol has a biocompatible and flexible polyether backbone with a high number of hydrophilic functional groups, which increases polyglycerol versatility and enables the production of hydrogels [14]. Citric acid is a relatively small and multifunctional monomer with pendant functional groups that allow future ester bond-crosslink and hydrogen bonding $[15,16]$. Fatty acids are relative long monomers with a carboxylic functional group that with chemical modification of doubledouble bond of unsaturated fatty acids allows the formation of crosslinked polymer structures [17]. The present study focuses on the synthesis and characterization of novel polymeric materials from biodegradable monomers that respond to $\mathrm{pH}$ and temperature stimuli. The effect of type, amount, and size of crosslinking agents on thermal, mechanical, and stimuli-responsive properties was investigated.

Previous studies have reported the synthesis of hydrogels by crosslinking glycerol-derived polyglycerol with 
TABLE 1: Composition of fatty acids used as crosslinking agent. Characterization was made using a gas chromatography system (Agilent Technologies 6890 series), coupled to a FID detector, using an Agilent DB23 column and SUPELCO 38 FAMES as standards.

\begin{tabular}{lc}
\hline Fatty acid & Area percentage (\%) \\
\hline Palmitic & 9.08 \\
Stearic & 8.12 \\
Oleic & 32.14 \\
Linoleic & 36.31 \\
Linolenic & 3.56 \\
Eicosenoic & 4.06 \\
Others & 6.71 \\
\hline
\end{tabular}

poly(ethylene glycol) diglycidyl ether PEGDE [14]. The resulting hydrogels exhibited $\mathrm{pH}$-dependent swelling behavior with a higher swelling capability at acidic $\mathrm{pH}$ value, compared with swelling at neutral and basic $\mathrm{pH}$ values. Significant research has been focused on materials that change their properties in response to external physical and chemical stimuli such as $\mathrm{pH}$, electric field, temperature, and ionic strength of the swelling agent, due to acid or basic pendant functional groups present on the polymer backbone [18-21]. Citric acid has been used as crosslinking agent for hydrogels production, for instance, with poly(vinyl alcohol) [22], with varying glycol unit (ethylene glycol, diethylene glycol, and triethylene glycol) [23], and with cellulose [24]. Fatty acids, such as linoleic and oleic acid, have been traditionally used to improve mechanical properties and chemical resistance in polymeric materials $[25,26]$.

Formation of hydrogels was confirmed using FTIR. Pendant acidic functional groups were identified in the polymer network which either accepts or releases protons as result of changing external $\mathrm{pH}$. The calorimetric analysis was performed to study the effect of amount and size of crosslinking agents on glass transition temperature. Creep-recovery tests were conducted to elucidate structure-property relationship in mechanical properties. Hydrogels swelling behavior was determined at different $\mathrm{pH}$ and temperatures. Finally, SEM micrographs were taken to study morphological properties.

\section{Materials and Methods}

2.1. Materials. Glycerol (85\%) and Sulfuric acid (95\%) were obtained from Merck. Citric acid (99\%) is a commercial product of Suquin Ltda., Bucaramanga, Co. The mixture of fatty acids used in this study was purchased from Laboratorios León S.A., Bucaramanga, Co. Its composition is listed in Table 1.

\subsection{Experimental Procedure}

2.2.1. Hydrogels Synthesis. Hydrogels synthesis was carried out in two steps using the same reaction system: first step, polymerization reaction of glycerol to produce polyglycerol, and second step, crosslinking reaction of polyglycerol; see Figure 1. The reaction system consisted in a $50 \mathrm{~mL}$ glass

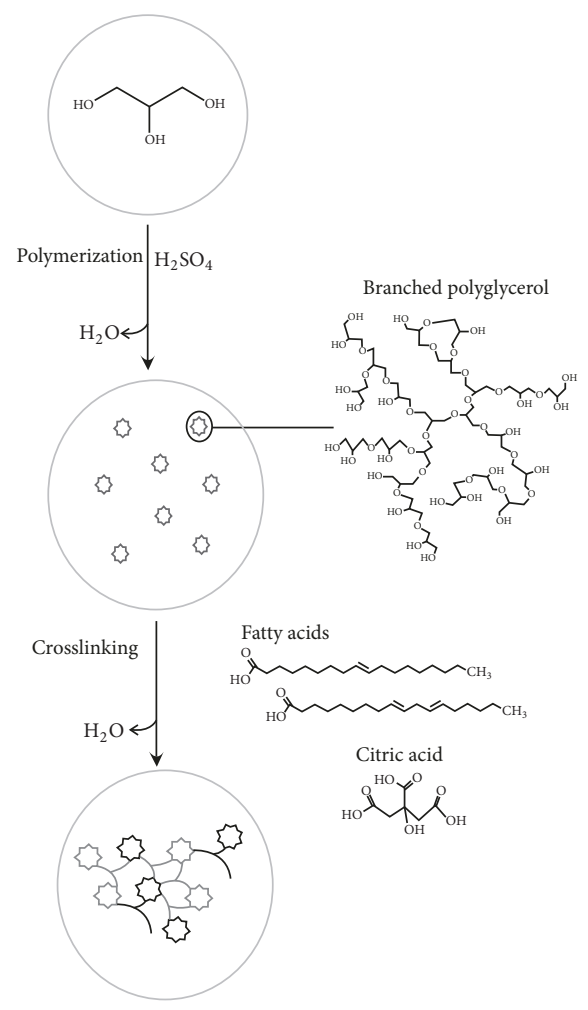

FIGURE 1: Schematic representation of hydrogel synthesis. First, the glycerol polymerization and the subsequent addition of crosslinking agents.

reactor equipped with a nitrogen inlet, catalyst feeding, thermometer inlet, and a distillation trap to continuously remove water from the reaction mixture. The temperature was maintained at $160^{\circ} \mathrm{C}$ using a temperature-controlled heating bath. A vacuum pump was attached to the reactor through the condenser. Condensation reactions were carried out at pressure of 22 in Hg. $4.8 \%$ w/w of Sulfuric acid was used as a catalyst [13]. The crosslinking agents were added to the reaction mass of polymerized glycerol, just before reaching the gel point, without further addition of catalyst. The reaction proceeded until the hydrogel reached the gel point.

Three different hydrogels were synthesized changing the nature of crosslinking agent: a hydrogel with citric acid as crosslinking agent, a hydrogel with fatty acids as crosslinking agent, and a hydrogel with both citric and fatty acids as crosslinking agent. Molar ratio between polyglycerol hydroxyl groups and crosslinking agents carboxyl groups and molar composition of crosslinking agents of synthesized hydrogels are reported in Table 2. After polymerization and crosslinking process, the hydrogels were washed with distilled water to remove catalyst and unreacted monomers.

2.3. Characterization. The synthesized hydrogels were tested to determinate their swelling behavior as a function of time in distilled water at room conditions. Absorption measurements were also made at $35^{\circ} \mathrm{C}, 55^{\circ} \mathrm{C}$, and $85^{\circ} \mathrm{C}$ and $\mathrm{pH} 4,7$, and 10 to establish if the synthesized hydrogels have a response to temperature and $\mathrm{pH}$. The absorption tests at different $\mathrm{pH}$ 
TABLE 2: Samples compositions used for the hydrogels synthesis.

\begin{tabular}{lcc}
\hline Hydrogel & $\begin{array}{c}\text { Molar ratios of OH } \\
\text { groups of polyglycerol } \\
(\text { PG) : COOH groups of } \\
\text { crosslinking agents (CG) }\end{array}$ & $\begin{array}{c}\text { Molar compositions of } \\
\text { COOH groups of citric } \\
\text { acid (CA) and fatty acids } \\
\text { (FFAs) }\end{array}$ \\
\hline PG-FFAs & $1: 0.05$ & $100 \%$ FFAs \\
PG-CA & $1: 0.45$ & $100 \%$ CA \\
PG-(CA, & $1: 0.5$ & $90 \%$ CA-10\% FFAs \\
FFAs) & & \\
\hline
\end{tabular}

were performed using Hanna Instruments buffer solutions. All measurements were done in triplicate. The hydrogels water absorption was calculated using (1) [14].

$$
\% S=\frac{W_{s}-W_{d}}{W_{d}} * 100 \%,
$$

where $W_{d}$ is the dry hydrogel weight and $W_{s}$ is the swollen hydrogel weight.

Fourier Transform Infrared Spectroscopy (FTIR) was used to identify functional groups in synthesized hydrogels. The infrared spectra were obtained in transmittance mode in a Thermo Scientific spectrometer (Nicolet 1550 FTIR). Glass transition temperatures were obtained using Differential Scanning Calorimetry (DSC), and measurements were carried out on a DSC Discovery, TA Instruments, Inc. (USA). The samples were subjected to the following thermal schedule: first heating from $-80^{\circ} \mathrm{C}$ to $180^{\circ} \mathrm{C}$ at $5^{\circ} \mathrm{C} / \mathrm{min}$ to eliminate volatile substances and thermal history of the materials; second, a cooling from $180^{\circ} \mathrm{C}$ to $-90^{\circ} \mathrm{C}$ at $10^{\circ} \mathrm{C} / \mathrm{min}$; third, final heating from $-90^{\circ} \mathrm{C}$ to $200^{\circ} \mathrm{C}$. All scans were performed under nitrogen purge gas of $50 \mathrm{~mL} / \mathrm{min}$. Scanning Electron Microscopy (SEM) measurements were performed to study the morphology of synthesized hydrogels. Samples were pretreated by being fully swollen in distilled water, then frozen in liquid nitrogen, and lyophilized for $72 \mathrm{~h}$. Freeze-dried hydrogels were consequently fractured for morphology visualization. The uncoated environmental SEM images were taken using a Quanta FEG 650 at acceleration voltages of $15 \mathrm{kV}$. Creep-recovery tests of synthesized hydrogels were tested using Dynamic Mechanical Analysis (DMA), and measurements were carried out on a Q800 dynamic mechanical analyzer, TA Instruments Inc. (USA). The samples were swollen and tests were carried out in water medium. Treatments were compressed at $0.007 \mathrm{MPa}$ during $20 \mathrm{~min}$ at three different temperatures; after that, the samples were recovered for $20 \mathrm{~min}$.

\section{Results and Discussion}

3.1. FTIR Spectral Studies of Polymeric Hydrogels. Fourier Transform Infrared Spectroscopy (FTIR) analysis was conducted to synthesized hydrogels and compared with polyglycerol spectra; see Figure 2. The hydrogels and polyglycerol spectra show hydroxyl group band from 3050 to $3600 \mathrm{~cm}^{-1}$ indicative of alcohol groups. Hydrogels $\mathrm{O}-\mathrm{H}$ stretch exhibits a loss of intensity in comparison with polyglycerol as a consequence of crosslinking linkages. The $\mathrm{C}-\mathrm{H}$ stretching band was

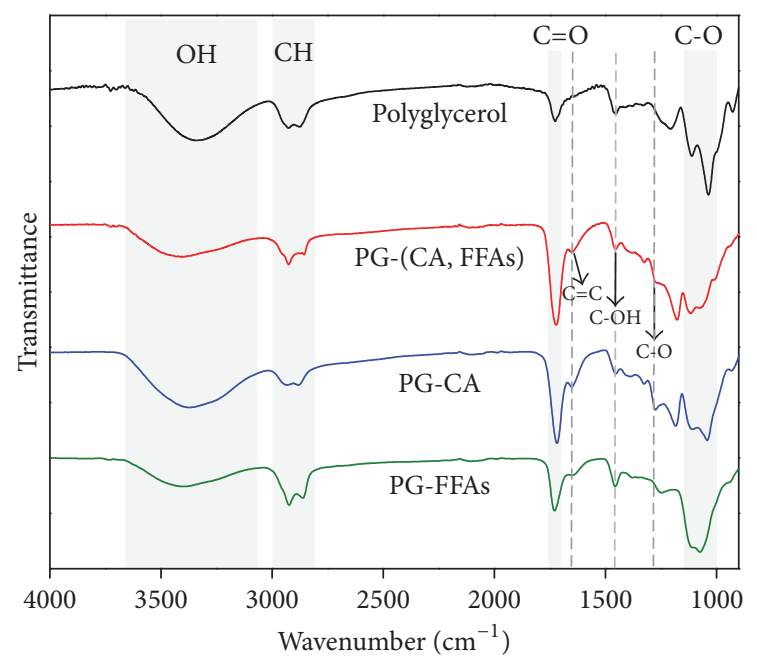

FIGURE 2: FTIR spectra of glycerol-derived polyglycerol and polyglycerol-based hydrogels. The main peaks associated with the structures are highlighted.

observed from 2800 to $3000 \mathrm{~cm}^{-1}$ [27]. Hydrogels with fatty acids as crosslinking agents, treatment PG-(CA, FFAs), and treatment PG-FFAs show well-defined C-H stretching as a consequence of hydrocarbon chains of fatty acids. Absorption in the range of $1700-1750 \mathrm{~cm}^{-1}$ in hydrogels spectrum is related to $\mathrm{C}=\mathrm{O}$ stretch of aliphatic esters produced by esterification reactions between polyglycerol hydroxyl groups with carboxylic groups present in citric acid and fatty acids. In the specific case of polyglycerol, this absorption is due to acrolein formation [28]. Absorption at $1650 \mathrm{~cm}^{-1}$ is associated with $\mathrm{C}=\mathrm{C}$ group of both unsaturated fatty acids and undesired products such as acrolein.

The spectra showed a peak at $1455 \mathrm{~cm}^{-1}$ that corresponds to $\mathrm{C}-\mathrm{OH}$ in-plane bending and $\mathrm{CH}_{2}$ bending [28]. The peaks appearing at $1235-1290 \mathrm{~cm}^{-1}$ in hydrogels spectrum are related to $\mathrm{C}-\mathrm{O}$ vibration of unreacted carboxylic groups. These pendant acidic functional groups will play a crucial role in stimuli-responsive properties. The $\mathrm{C}$-O vibration of unreacted carboxylic groups is highlighted in Figure 3. It is observed that hydrogel PG-FFAs exhibits lower C-O vibration intensity in comparison with hydrogels that have citric acid as crosslinking agent. This result is due to citric acid structure, which has three carboxylic functional groups, and not all of them react in the crosslink reaction. Finally, absorption at $1000-1150 \mathrm{~cm}^{-1}$ is related to C-O stretching of the ether groups present in the polyglycerol backbone [14] and etherification reactions between polyglycerol and crosslinking agents. Polyglycerol hydroxyl groups can react by etherification reactions with citric acid hydroxyl group and with fatty acids hydrated carbon-carbon double bonds.

3.2. Thermal Properties. Treatment PG-FFAs exhibits the lowest glass transition at $40.76^{\circ} \mathrm{C}$ (Figure 4), which is related to the lowest crosslink density and long chain size of fatty acids used as crosslinking agent. Therefore, less temperature is needed to change the hydrogel state from glassy to rubbery. 


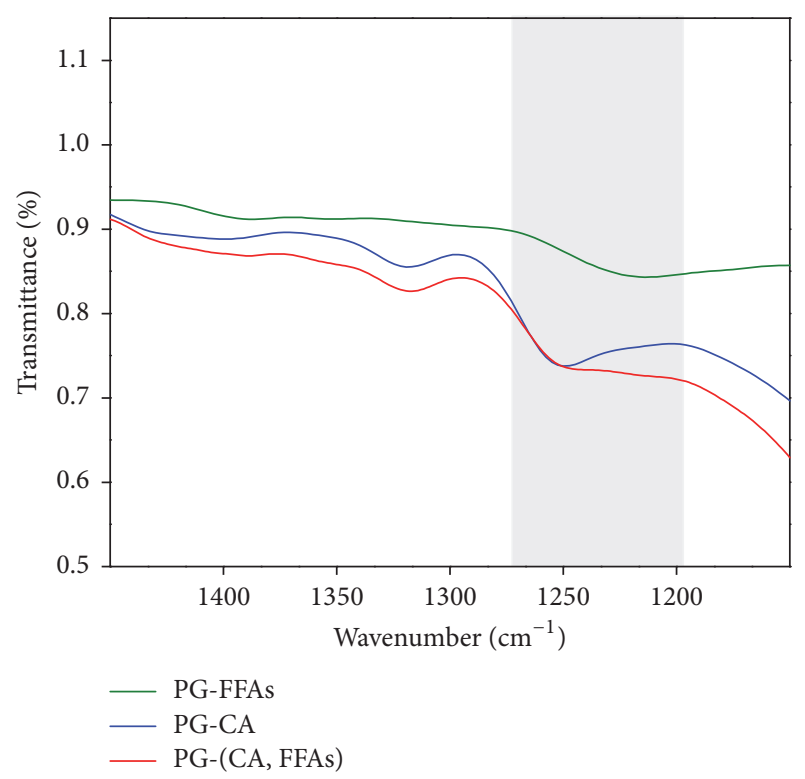

FIGURE 3: Zoom of the FTIR spectra at $1235-1290 \mathrm{~cm}^{-1}$ of the three synthesized hydrogels. The highlighted $\mathrm{C}-\mathrm{O}$ vibration is related to the unreacted carboxylic groups.

On the other hand, treatment PG-CA exhibits the highest glass transition at $50.70^{\circ} \mathrm{C}$. This result is due to higher crosslink density and citric acid short chain, which gives less flexibility to the material. In the particular case of hydrogel PG-(CA, FFAs), this material exhibits a glass transition temperature at $42.87^{\circ} \mathrm{C}$, which is between the two glass transitions just described. This result can be explained by the combined features of each used crosslinking agent.

3.3. Mechanical Properties. The adequacy of hydrogels for specific applications depends on their mechanical properties and response times [29]. In this work, mechanical properties were evaluated by a creep-recovery test at different temperatures: $35^{\circ} \mathrm{C}, 55^{\circ} \mathrm{C}$, and $70^{\circ} \mathrm{C}$. Tests were performed in water medium simulating a typical condition of hydrogels in their applications. Results are shown in Figure 5. In general, all hydrogels have a small transition before a flat equilibrium region. Hydrogels deformation and percentage of strain recovery (SR) at different temperatures depend strongly on crosslink density, size, and type of crosslink agent. PG-FFAs present the highest deformation at all temperatures. This hydrogel has a slight variation of deformation at $35^{\circ} \mathrm{C}$ and $55^{\circ} \mathrm{C}$ and a significant increase in deformation at $70^{\circ} \mathrm{C}$ due to high molecular mobility at temperatures above glass transition temperature. Percentages of recovery of PG-FFAs are relatively low, $38.41,41.45 \%$, and $27.55 \%$ at $35^{\circ} \mathrm{C}, 55^{\circ} \mathrm{C}$, and $70^{\circ} \mathrm{C}$, respectively. High deformations and relatively low percentages of recovery of PG-FFAs hydrogel are due to low crosslink density and long chains of fatty acids used to form the network. High crosslink density and short chains of citric acid resulted in PG-CA hydrogels with relatively low deformation related to PG-FFA hydrogel. PG-CA hydrogel presented an increase in deformation and decrease in the

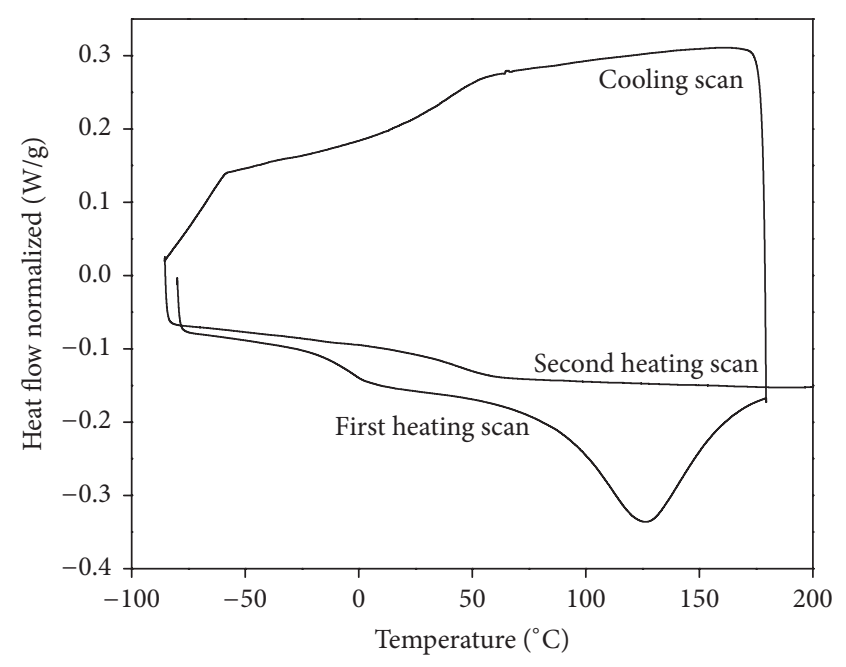

(a)

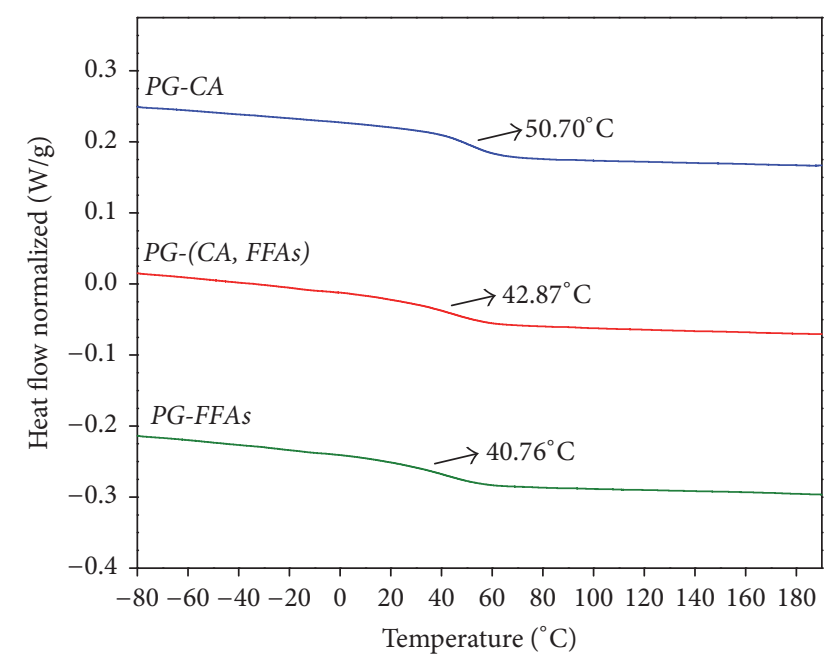

(b)

FIGURE 4: (a) First and second heating scan DSC result for hydrogels synthesized. (b) Hydrogels glass transition temperatures found in the second heating scan.

percentage of recovery as temperature increases. This result may be related to hydrogel chains mobility since glass transition temperature is $50^{\circ} \mathrm{C}$. Finally, PG-(CA, FFAs) hydrogel has above $55^{\circ} \mathrm{C}$ the lowest deformation and the highest percentage of recovery of all hydrogels at this temperature.

3.4. Swelling Properties. The hydrogel with the highest swelling degree is PG-(CA, FFAs). It absorbs more than seven times its own weight; see Figure 6 . This swelling behavior may be related to the contribution of both crosslinking agents; fatty acids long chains may contribute to large pore sizes, and unreacted hydroxyl groups of citric acid in addition to unreacted polyglycerol hydroxyl groups increase water affinity. Hydrogel PG-CA probably has smaller pore sizes for holding water caused by citric acid short chains. Finally, hydrogel PG-FFAS has only fatty acids that, despite its long 


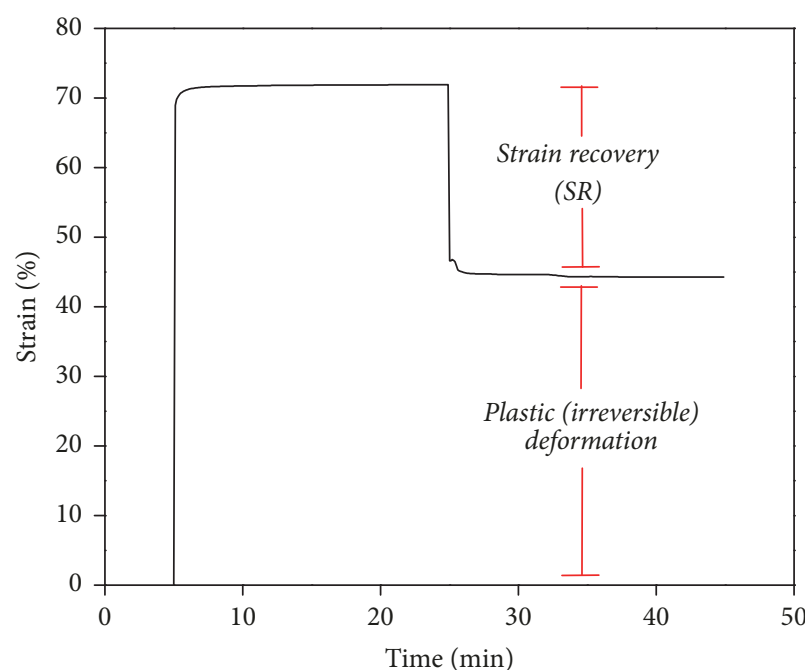

(a)

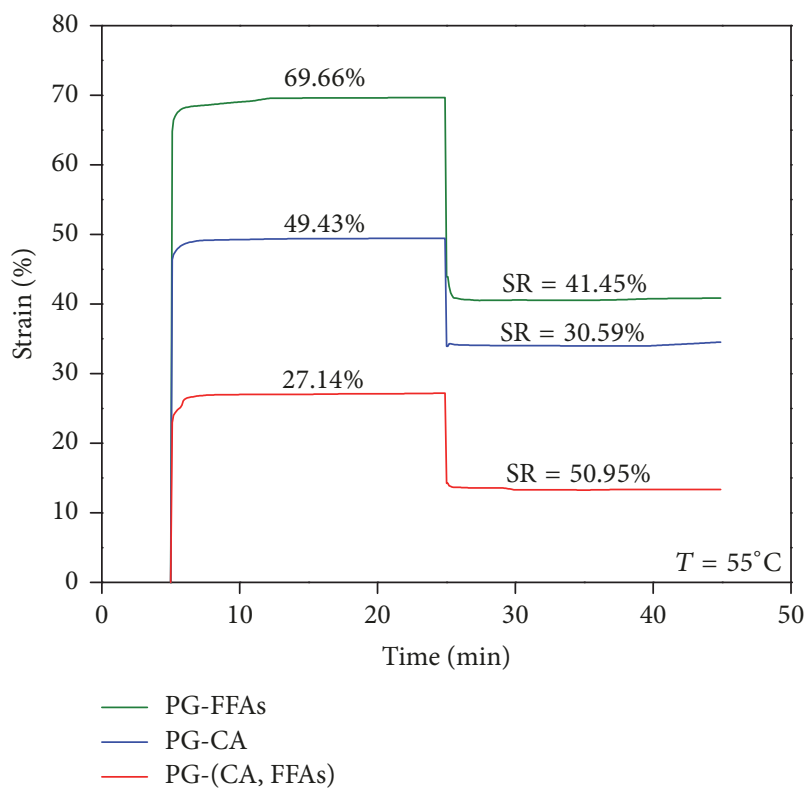

(c)

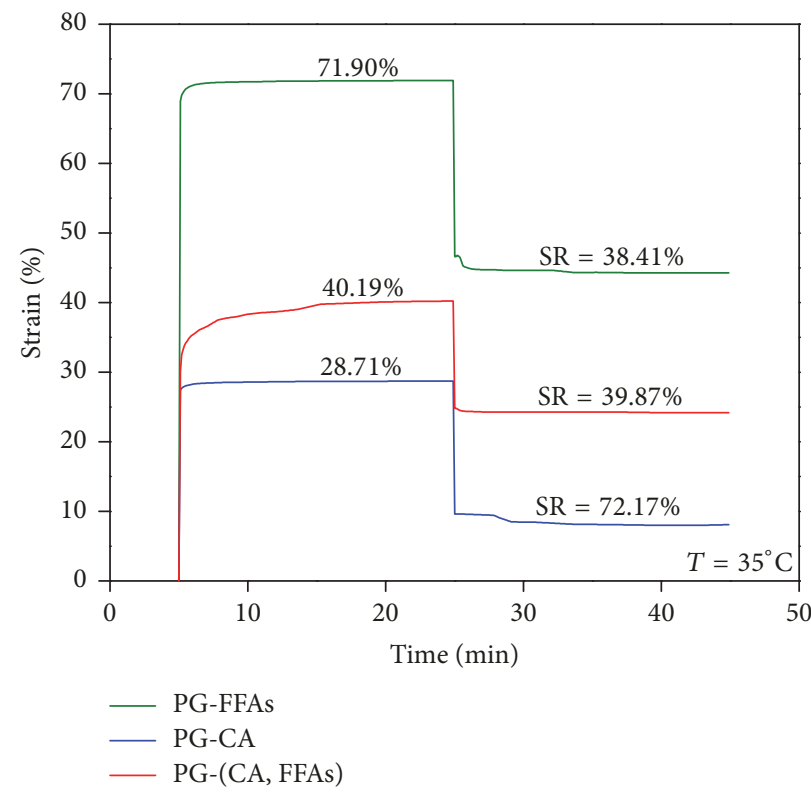

(b)

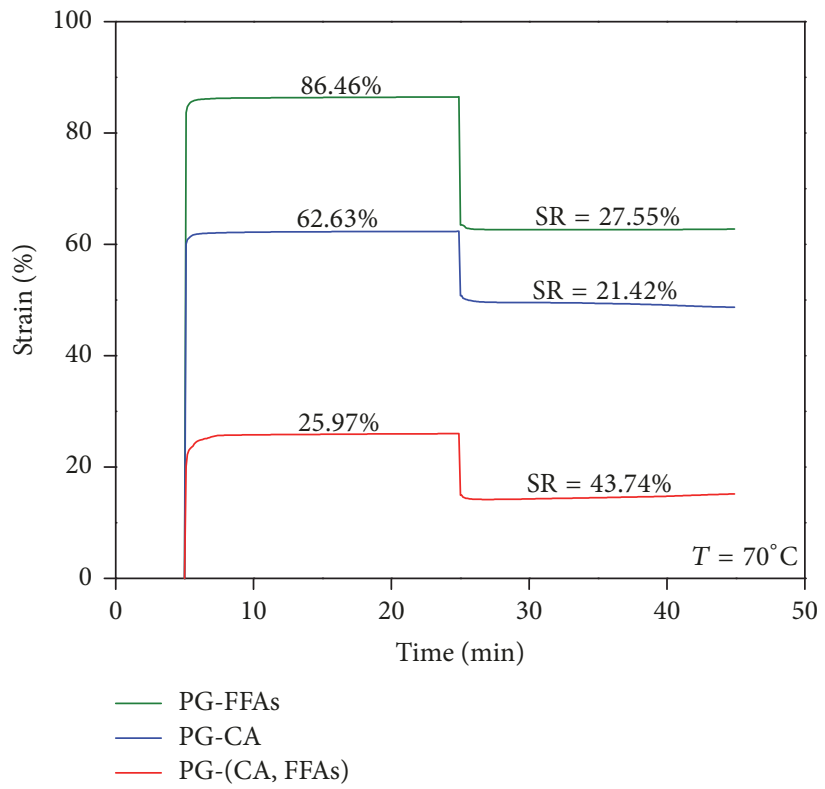

(d)

FIGURE 5: Submersion-compression creep for treatments PG-FFAs, PG-CA, and PG-(CA, FFAs) in DMA at $35^{\circ} \mathrm{C}, 55^{\circ} \mathrm{C}$, and $70^{\circ} \mathrm{C}$.

chain size, its crosslinking degree is low holding limited water molecules within its structure.

3.4.1. Effect of $p H$ on Swelling Behavior. The swelling behavior of hydrogels was studied after 60 minutes of water absorption at $\mathrm{pH} 4,5,7$, and 10. From the results, it was concluded that the hydrogels swelling behavior depends on external $\mathrm{pH}$; see Figure 7. The greater swelling capability of hydrogel PG-(CA, FFAs) is observed when the $\mathrm{pH}$ value is 10 , in which the hydrogel absorbs more than 13.7 times its own weight. At $\mathrm{pH} 4$ and 7, the hydrogel also increases its swelling capability absorbing 8.6 and 10 times their own weights, respectively.
As reported in other studies [20,30], an explanation for these results is that at basic $\mathrm{pH}$ values, the unreacted carboxylic groups become ionized producing carboxylate ions $\left(\mathrm{RCOO}^{-}\right)$, and $\mathrm{H}^{+}$combines with $\mathrm{OH}^{-}$from basic solution forming $\mathrm{H}_{2} \mathrm{O}$. Negative charges of carboxylate ions create an electrostatic repulsion between the polymer chains forcing the hydrogel to uncoil, increasing free volumes in the hydrogel network.

In the specific case of treatment PG-FFAs, its swelling degree at high $\mathrm{pH}$ is lower in comparison with the other two treatments because this material presents less unreacted carboxylic groups; see Figure 3. A different trend result was 


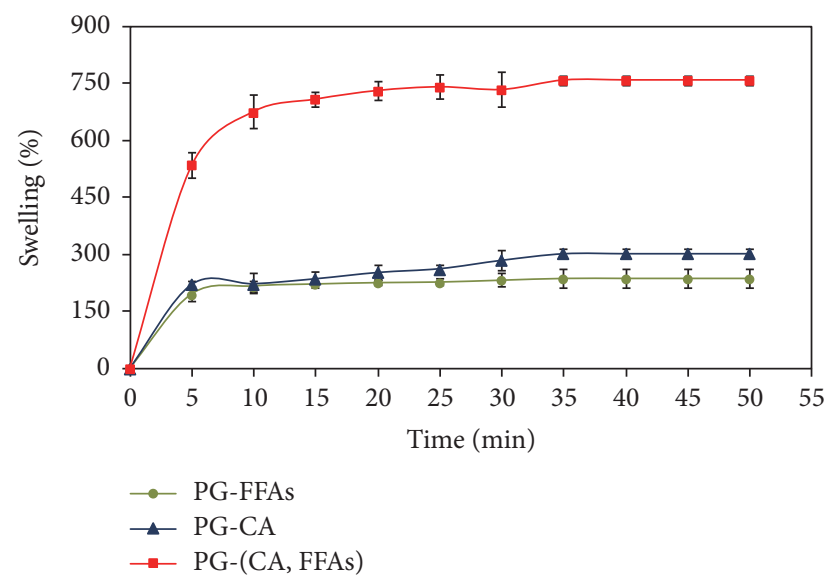

Figure 6: Swelling behavior of treatments PG-FFAs (๑), PG-CA $(\boldsymbol{\Delta})$, and PG-(CA, FFAs) (घ). Measurements were taken at $20^{\circ} \mathrm{C}$ and pH 5 using (1).

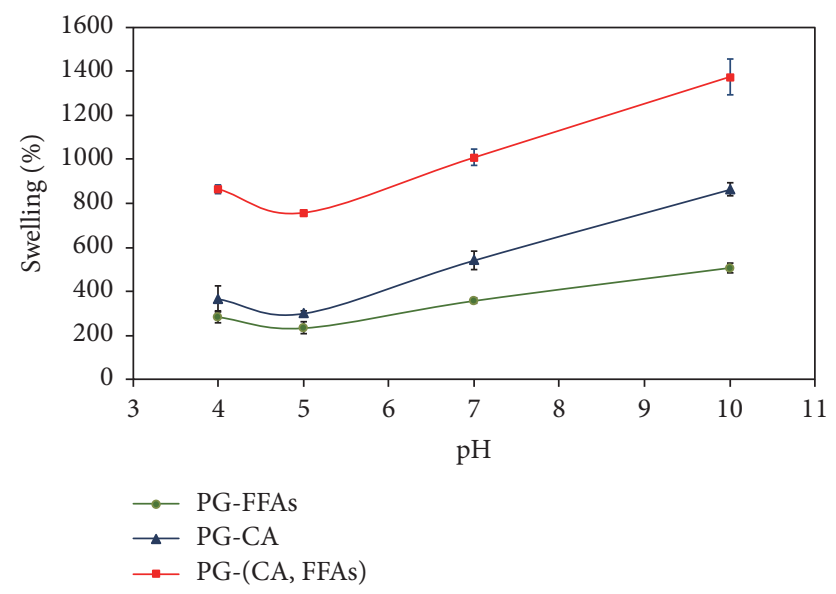

FIGURE 7: pH-dependent swelling behavior of treatments PG-FFAs

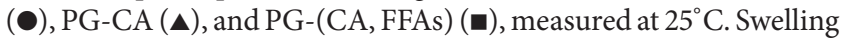
percentage was calculated using (1).

obtained at $\mathrm{pH} 4$ where hydrogels showed higher swelling behavior than that at $\mathrm{pH} 5$; this can be related to the attraction of $\mathrm{H}^{+}$ions to hydroxyl and ether groups, as reported before [14]. Swelling results at different $\mathrm{pH}$ correlate with unreacted carboxylic groups reported previously in FTIR results; see Figure 3; hydrogels with more free unreacted carboxylic groups have a greater response to changes in $\mathrm{pH}$.

3.4.2. Effect of Temperature on Swelling Behavior. Hydrogels swelling behavior was studied after 60 minutes of water absorption at $20,35,55$, and $85^{\circ} \mathrm{C}$ (Figure 8). Results show that at $35^{\circ} \mathrm{C}$ the three hydrogels present the lowest swelling degree. At this temperature, all hydrogels are below glass transition temperature as shown in Figure 4. At temperatures above $55^{\circ} \mathrm{C}$, all hydrogels are above glass transition temperature. As a result, hydrogels chain mobility increases water absorption. The greater swelling capability is observed at $85^{\circ} \mathrm{C}$ where the PG-(CA, FFAs) hydrogel absorbs water 8.5 times its own weight.

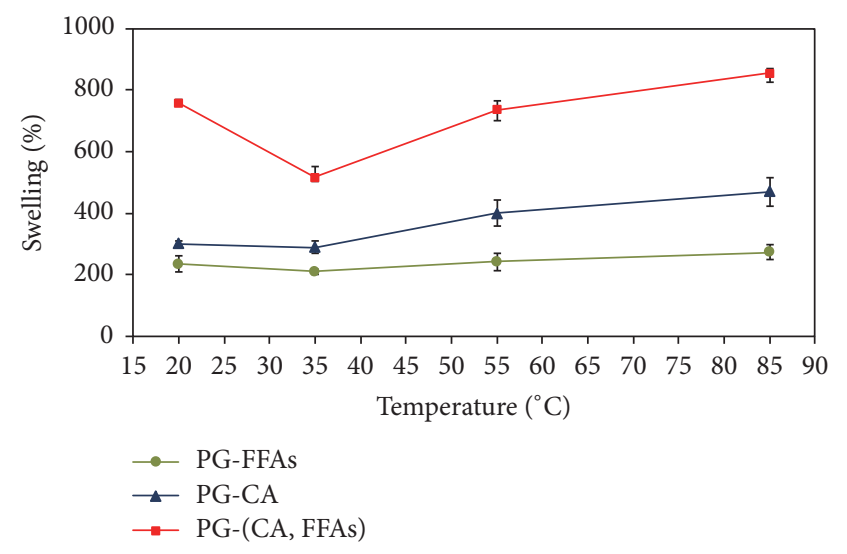

FIGURE 8: The temperature-dependent swelling behavior of treat-

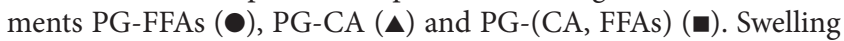
percentage was calculated using (1).

3.5. Morphological Properties. Morphology of hydrogel PG(CA, FFAs) is shown in Figure 9. It was found that the hydrogel exhibits an uneven, rough, heterogeneous, and slightly porous structure. The observed hydrogel pore heterogeneity (pore diameters from 2 to $62 \mu \mathrm{m}$ ) could be related to the randomness of crosslinking reactions between polyglycerol and citric-fatty acids which have different chain lengths and functional groups. Furthermore, the porous structure has interconnected pores forming open channels for capillary absorption of water; see Figure 9(b).

This material looks like a sponge and its swelling process follows the same principle, keeping the water within its structure due to its free volumes. This hydrogel may be considered as macroporous material according to IUPAC [31].

\section{Conclusions}

The effect of type, amount, and size of crosslinking molecules such as citric and fatty acids on thermal, mechanical, swelling, and stimuli-responsive polyglycerol-based hydrogel properties was studied. The small citric acid molecule used as crosslinking agent produced high crosslink density which manifested itself in higher glass transition temperature, relatively low hydrogel deformation, and higher amount of unreacted carboxylic functional groups that propitiated stimuliresponsive properties. On the other hand, large fatty acids molecules used as crosslinking agent produced low crosslink density which manifested itself in lower glass transition temperature, higher hydrogen deformation, and less unreacted carboxylic functional groups. It was concluded that it is possible to tune hydrogel properties by the judicial combination of citric and fatty acids crosslinking agents. The resulted hydrogel combining both crosslink agents has an intermediate glass transition temperature, relative short deformation with high recovery, and unreacted carboxylic functional groups that improved stimuli-responsive properties in comparison with hydrogels with uncombined crosslinking agents. 


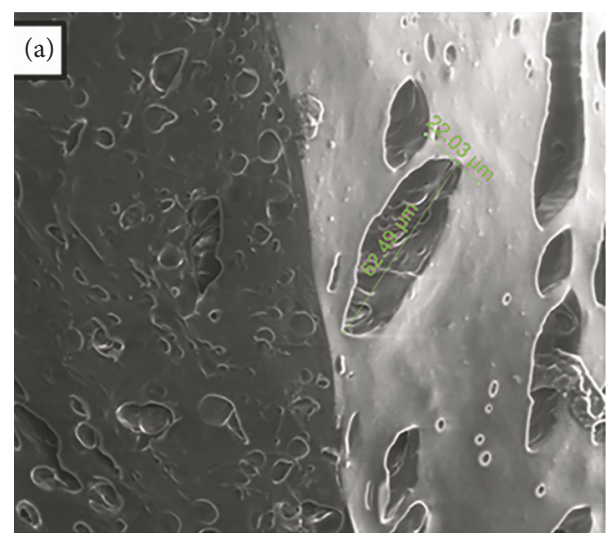

(a)

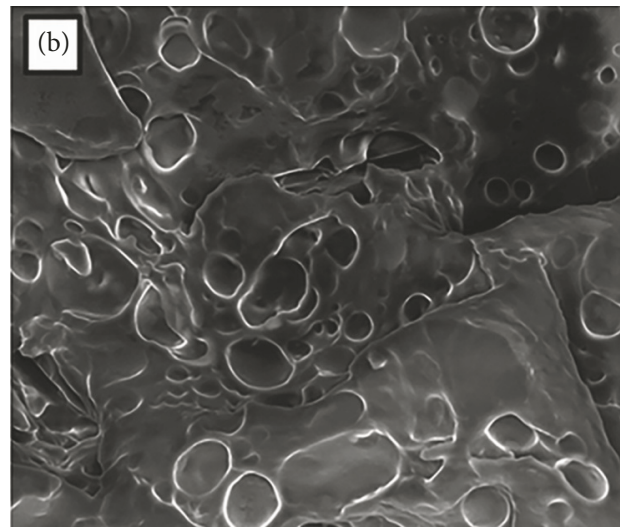

(b)

Figure 9: SEM micrographs of the hydrogel PG-(CA, FFAs).

\section{Conflicts of Interest}

The authors declare that they do not have any conflicts of interest.

\section{Acknowledgments}

This work was supported by Vicerrectoría de Investigación $y$ Extension at Universidad Industrial de Santander (UIS) and funded by the agreement of cooperation (Code 9460) between UIS, Corasfaltos, and Colciencias. Scanning Electron Microscopy (SEM) measurements were supported by Laboratory of Microscopy of Parque Tecnológico Guatiguará (PTG) UIS. The authors thank Dr. Álvaro Ramírez for reviewing this paper.

\section{References}

[1] A. Słoniewska and B. Pałys, "Supramolecular polyaniline hydrogel as a support for urease," Electrochimica Acta, vol. 126, pp. 90-97, 2014.

[2] E. S. Dragan, "Design and applications of interpenetrating polymer network hydrogels. A review," Chemical Engineering Journal, vol. 243, pp. 572-590, 2014.

[3] L. Bédouet, F. Pascale, L. Moine et al., "Intra-articular fate of degradable poly(ethyleneglycol)-hydrogel microspheres as carriers for sustained drug delivery," International Journal of Pharmaceutics, vol. 456, no. 2, pp. 536-544, 2013.

[4] D. S. Jones, G. P. Andrews, D. L. Caldwell, C. Lorimer, S. P. Gorman, and C. P. McCoy, "Novel semi-interpenetrating hydrogel networks with enhanced mechanical properties and thermoresponsive engineered drug delivery, designed as bioactive endotracheal tube biomaterials," European Journal of Pharmaceutics and Biopharmaceutics, vol. 82, no. 3, pp. 563-571, 2012.

[5] Y. Liang and K. L. Kiick, "Heparin-functionalized polymeric biomaterials in tissue engineering and drug delivery applications," Acta Biomaterialia, vol. 10, no. 4, pp. 1588-1600, 2014.

[6] N. K. Singh and D. S. Lee, "In situ gelling pH- and temperaturesensitive biodegradable block copolymer hydrogels for drug delivery," Journal of Controlled Release, vol. 193, pp. 214-227, 2014.

[7] A. I. Raafat, M. Eid, and M. B. El-Arnaouty, "Radiation synthesis of superabsorbent CMC based hydrogels for agriculture applications," Nuclear Instruments and Methods in Physics Research Section B: Beam Interactions with Materials and Atoms, vol. 283, pp. 71-76, 2012.

[8] C. Demitri, F. Scalera, M. Madaghiele, A. Sannino, and A. Maffezzoli, "Potential of cellulose-based superabsorbent hydrogels as water reservoir in agriculture," International Journal of Polymer Science, vol. 2013, Article ID 435073, 6 pages, 2013.

[9] X. Ma, Y. Li, W. Wang, Q. Ji, and Y. Xia, “Temperature-sensitive poly(N-isopropylacrylamide)/graphene oxide nanocomposite hydrogels by in situ polymerization with improved swelling capability and mechanical behavior," European Polymer Journal, vol. 49, no. 2, pp. 389-396, 2013.

[10] S. Saber-Samandari, S. Saber-Samandari, and M. Gazi, "Cellulose-graft-polyacrylamide/hydroxyapatite composite hydrogel with possible application in removal of $\mathrm{Cu}$ (II) ions," Reactive and Functional Polymers, vol. 73, no. 11, pp. 1523-1530, 2013.

[11] T. Endo, R. Ikeda, Y. Yanagida, and T. Hatsuzawa, "Stimuliresponsive hydrogel-silver nanoparticles composite for development of localized surface plasmon resonance-based optical biosensor," Analytica Chimica Acta, vol. 611, no. 2, pp. 205-211, 2008.

[12] M. Bahram, F. Hoseinzadeh, K. Farhadi, M. Saadat, P. NajafiMoghaddam, and A. Afkhami, "Synthesis of gold nanoparticles using $\mathrm{pH}$-sensitive hydrogel and its application for colorimetric determination of acetaminophen, ascorbic acid and folic acid," Colloids and Surfaces A: Physicochemical and Engineering Aspects, vol. 441, pp. 517-524, 2014.

[13] C. Ardila-Suárez, D. Rojas-Avellaneda, and G. E. RamirezCaballero, "Effect of Temperature and Catalyst Concentration on Polyglycerol during Synthesis," International Journal of Polymer Science, vol. 2015, Article ID 910249, 8 pages, 2015.

[14] S. Salehpour, C. J. Zuliani, and M. A. Dubé, "Synthesis of novel stimuli-responsive polyglycerol-based hydrogels," European Journal of Lipid Science and Technology, vol. 114, no. 1, pp. 92-99, 2012.

[15] R. T. Tran, Y. Zhang, D. Gyawali, and J. Yang, "Recent developments on citric acid derived biodegradable elastomers," Recent 
Patents on Biomedical Engineering, vol. 2, no. 3, pp. 216-227, 2009.

[16] D. Gyawali, P. Nair, Y. Zhang et al., "Citric acid-derived in situ crosslinkable biodegradable polymers for cell delivery," Biomaterials, vol. 31, no. 34, pp. 9092-9105, 2010.

[17] L. Fertier, H. Koleilat, M. Stemmelen et al., "The use of renewable feedstock in UV-curable materials-A new age for polymers and green chemistry," Progress in Polymer Science, vol. 38, no. 6, pp. 932-962, 2013.

[18] M. Hamidi, A. Azadi, and P. Rafiei, "Hydrogel nanoparticles in drug delivery," Advanced Drug Delivery Reviews, vol. 60, no. 15, pp. 1638-1649, 2008.

[19] H. Priya James, R. John, A. Alex, and K. Anoop, "Smart polymers for the controlled delivery of drugs - a concise overview," Acta Pharmaceutica Sinica B (APSB), vol. 4, no. 2, pp. 120-127, 2014.

[20] K. S. De, N. R. Aluru, B. Johnson, W. C. Crone, D. J. Beebe, and J. Moore, "Equilibrium swelling and kinetics of $\mathrm{pH}$-responsive hydrogels: models, experiments, and simulations," Journal of Microelectromechanical Systems, vol. 11, no. 5, pp. 544-555, 2002.

[21] T. Bartil, M. Bounekhel, C. Cedric, and R. Jeerome, "Swelling behavior and release properties of $\mathrm{pH}$-sensitive hydrogels based on methacrylic derivatives," Acta Pharmaceutica, vol. 57, no. 3, pp. 301-314, 2007.

[22] Z. Ajji, "Preparation of poly(vinyl alcohol) hydrogels containing citric or succinic acid using gamma radiation," Radiation Physics and Chemistry, vol. 74, no. 1, pp. 36-41, 2005.

[23] D. S. Franklin and S. Guhanathan, "Synthesis and characterization of citric acid-based pH-sensitive biopolymeric hydrogels," Polymer Bulletin, vol. 71, no. 1, pp. 93-110, 2014.

[24] C. Demitri, R. Del Sole, F. Scalera et al., "Novel superabsorbent cellulose-based hydrogels crosslinked with citric acid," Journal of Applied Polymer Science, vol. 110, no. 4, pp. 2453-2460, 2008.

[25] G. Lligadas, J. C. Ronda, M. Galià, and V. Cádiz, "Oleic and undecylenic acids as renewable feedstocks in the synthesis of polyols and polyurethanes," Polymer, vol. 2, no. 4, pp. 440-453, 2010.

[26] M. Moreno, M. Goikoetxea, and M. J. Barandiaran, "Biobasedwaterborne homopolymers from oleic acid derivatives," Journal of Polymer Science Part A: Polymer Chemistry, vol. 50, no. 22, pp. 4628-4637, 2012.

[27] H. S. Mansur, R. L. Oréfice, and A. A. P. Mansur, "Characterization of poly(vinyl alcohol)/poly(ethylene glycol) hydrogels and PVA-derived hybrids by small-angle X-ray scattering and FTIR spectroscopy," Polymer Journal, vol. 45, no. 21, pp. 7193-7202, 2004.

[28] S. Salehpour, Synthesis of stimuli-responsive hydrogels from glycerol, University of Ottawa, 2012.

[29] S. Abdurrahmanoglu and O. Okay, "Rheological behavior of polymer-clay nanocomposite hydrogels: Effect of nanoscale interactions," Journal of Applied Polymer Science, vol. 116, no. 4, pp. 2328-2335, 2010.

[30] J. Liu, Q. Li, Y. Su, Q. Yue, and B. Gao, "Characterization and swelling-deswelling properties of wheat straw cellulose based semi-IPNs hydrogel," Carbohydrate Polymers, vol. 107, no. 1, pp. 232-240, 2014.

[31] M. Thommes, K. Kaneko, A. V. Neimark et al., "Physisorption of gases, with special reference to the evaluation of surface area and pore size distribution (IUPAC Technical Report)," Pure and Applied Chemistry, vol. 87, no. 9-10, pp. 1051-1069, 2015. 


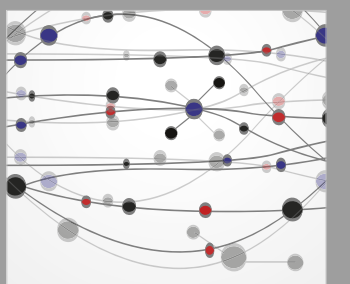

The Scientific World Journal
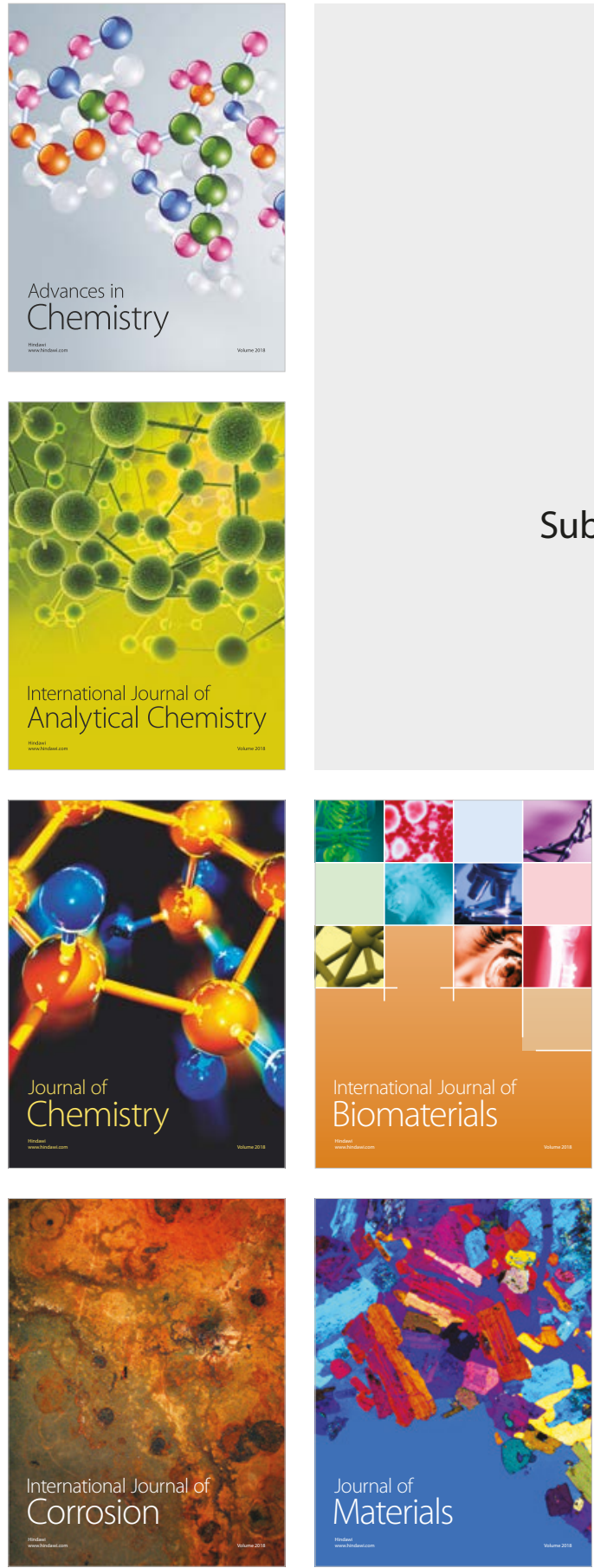

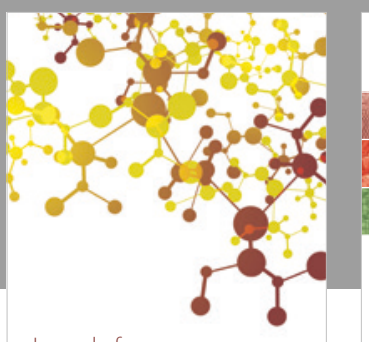

Journal of

Applied Chemistry
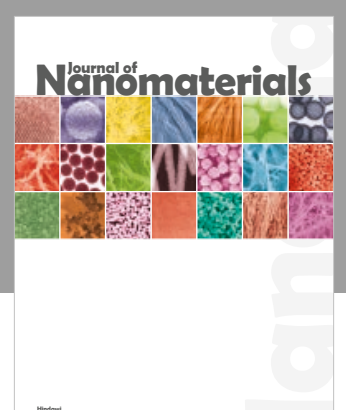

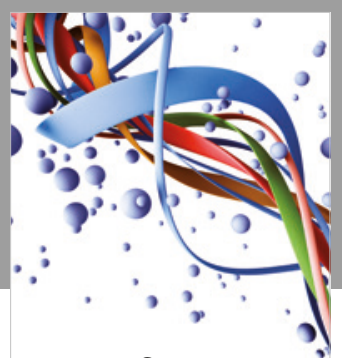

Scientifica

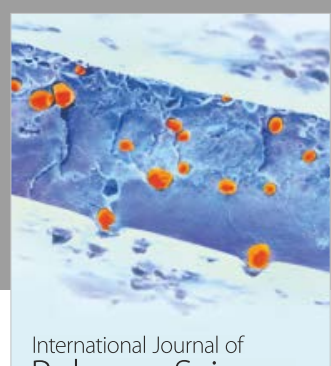

Polymer Science

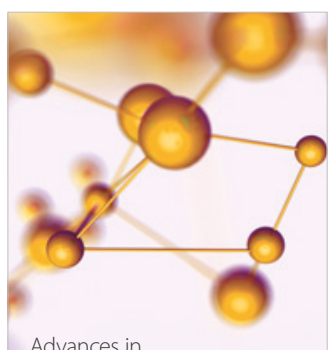

Physical Chemistry
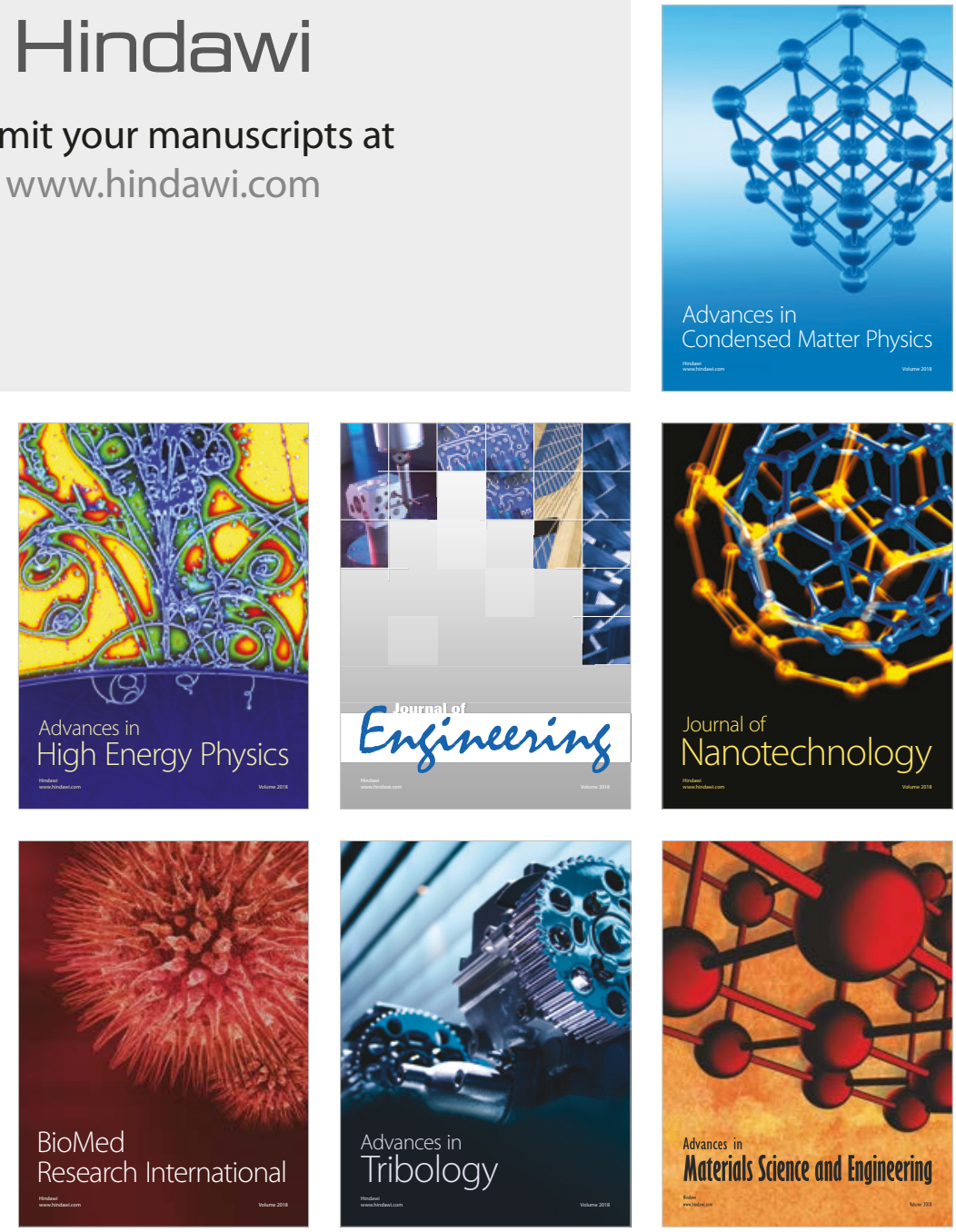